\title{
Designing a WSN Data Format for WLAN and Cellular Network Compatibility in an E-healthcare Framework
}

\author{
Soumyajit Giri \\ School of Computer \\ Engineering \\ KIIT University, Bhubaneswar, \\ Odisha, India
}

\author{
Kumar Debasis \\ School of Computer \\ Engineering \\ KIIT University, Bhubaneswar, \\ Odisha, India
}

\author{
Monideepa Roy \\ School of Computer \\ Engineering \\ KIIT University, Bhubaneswar, \\ Odisha,India
}

\begin{abstract}
The emergence of wireless sensor networks (WSNs) has powerful results on various areas of applications such as medicine and healthcare, air pollution detection, fire detection, water quality monitoring etc. Wireless biomedical sensor networks can supply non-obstructive, long-time, and real-time medical convoy for the chronic patients. The use of wireless medical sensor networks on a large scale can successfully minimize the maximum workloads for the technically growing society and prolonged health service for the chronically ill patients. It also reduces the possibility of errors caused by human. For this there is a need for a variety of types of data to be collected and sent to the sink for processing and diagnosis. In addition there may be heterogeneity in the types of devices which are participating in the network. Here author presents a Zigbee based Ehealthcare framework which will provide seamless support to patients from far off or rural areas. Here also proposed a data format to be captured in WSNs that will be compatible in WLANs as well as in the cellular networks which will collect data from the patients.
\end{abstract}

\section{General Terms}

Sensor Network, Clustering Technique, E-healthcare System.

\section{Keywords}

WSN, E-healthcare, T\&R Technique, Clustered Network, Zigbee.

\section{INTRODUCTION}

Wireless Sensor Networks (WSNs) can be widely deployed in area monitoring, environmental condition analyzing and monitoring (forest fire detection, landslide detection etc.), ehealthcare services, military, and many other areas. In most WSN monitoring systems, there is gathering and assembling of the sensor data (e.g., environmental monitoring data, health related patient data) for analysis, which is one of the most vital goals.

Author proposed a E-healthcare system of Remote Patient Monitoring where the idea is to mitigate the issues occurring in remote villages. In the present situation the ratio between chronic patient and doctor is very high. So, here the aim is to solve this problem with the help of E-healthcare system using WSN technology[4].The advantages are that it can be minimize the whole cost of the process, for both the patient and the companies who are providing the insurance facility and side by side making hi-tech healthcare available to the persons who require it. The Remote patient monitoring system revolves around a number of high-tech applications that employ sensor data for providing remote care for patients.
Through Wireless medical sensor networks patients will be under constant supervision. It will improve the quality of life of patients and decrease the probability of errors. Along with the health details of the patient it will also provide the information about the patient environment and photos/videos of the patient if needed. Here the Electrocardiogram (ECG) is one of the most widely used vital signals for monitoring and diagnosis of cardiac status as well as overall health condition. Hence, it is present in most of e-Healthcare monitoring systems. But, it requires high bandwidth requirements as compare as to other signals which measure temperature, heart rate, different gas saturation in blood etc. Wireless sensor network model includes Zigbee technology which helps transmitting signals for bio signals, medical imaging or video, EPR for electronic patient record, and AIV for audio (A) or image (I) or video (V) teleconferencing.

The method to deal with how the WSN data can be sent through a low power short-range ZigBee[11] technology in a format compatible with cellular and wireless technology. The ZigBee standard operates on the IEEE 802.15.4 physical radio specification. It is a modification of the Wireless Private Area Network (WPAN). This technology has some advantageous features like: 1) Transmission range of ZigBee is in between 10 to 100 meters. 2) Good for mobility equipment 3) Lowpower consumption, which means that the node goes into sleep mode when communication is not required. 4) Zigbee devices sustain their energy for a longer period of time as compared to other devices. 5) Less complexity, layer structure of ZigBee network is star, tree and mesh structure. 6) The scalability is high as it supports numerous nodes in a network. 7) The entry and removal of nodes in a network is very fast. 8) With higher bandwidth, and it can support multiple access which is suitable for wireless monitoring of health data in hospital environments.

The rest of the paper is summarized as follows: In section 2, the related work is discussed. Section 3 describes the proposed framework. Section 4 discusses about the transmit and receive technique used in the proposed framework. Finally in section 5 the conclusion and future work is given.

\section{RELATED WORK}

There are at present various techniques that have been proposed for efficient sensor data collection.

Guestrin et al. introduced BBQ [14] which calculates the sensor data for every query using estimated senor data. The sensor node which administers the model contacts the actual sensor node periodically and updates the model. Thus the 
communication traffic is reduced considerably. But accidental change in sensor data cannot be handled through this method.

Chu et al. introduced Ken [12], which describes about a technique which is used to gather information from sensor nodes called push type data collection method which is able to solve the issues that was associated with previously proposed technique Barbeque(BBQ which is pull type data collection technique). In the network every sensor node dispatches the sensed data within a fixed time interval to the control node which is present at the base station. However this method does not deal with reducing unnecessary transmission of data.

Madden et al. introduced a technique called TinyDB [8] which incorporates a query-processing technique. It deals with query languages such as Structured Query Language (SQL) to extract related data from several sensor nodes in the networks. But, this procedure has a difficulty when there are a huge number of nodes in the sensor network, the rate of collecting sensor information obviously decrease with respect to time. For this reason communication overhead increases for collecting sensor information from large scale network which is needed for analysing the environment.

Fujita et al. introduced the Broadcast and Collect (B\&C) [5], that gathers related information from various sensors nodes of the network. It is a data gathering procedure to distribute important information from the sensor nodes with the broadcast technique. That $\mathrm{B} \& \mathrm{C}$ technique broadcasts guessed information from sensor database and collects always real information of the sensor if the sensed information changes importantly from guessed sensor data reading. This technique has the limitation that when the sensor nodes are out of range from the gateway node.

In the next section author proposed an E-healthcare framework for providing healthcare to remote and rural areas using fast and lightweight data exchange.

\section{THE PROPOSED FRAMEWORK}

It is very difficult to constantly monitor patients in remote villages which are far away from health centres. Doctors may not be always available to supervise their health status. To solve these problems wireless sensor nodes can be used to monitor the health condition of patients and continuously relay the data to health centres located far away. Thus patients are under surveillance at all times, even when they are mobile. Sensors are small and light-weight devices that are portable enough to be carried anywhere without any discomfort. Thus wireless sensor nodes solve the issues in remote patient monitoring to a great extent.

Here all the sensor nodes attached to a body creates a network where all the sensor nodes sense the data and send their sensed data to their gateway node after that gateway node sends their data to the server. Here it presents a new technique called Transmit and Receive $(T \& R)$ using clustering method as shown Fig. 1 where the gateway node transmits the threshold data value to the sensor nodes present in the network and receives the data when it significantly differ from the transmitting data. Using this technique redundant data can be avoided coming from the sensor nodes. So, this proposal represents a helpful low energy consumption protocol for data gathering where the number of sensor nodes in wireless sensor network (WSN) is very high, and it will help to reduce the overhead of communication traffic and power usage. The proposed framework consists of the following steps:

Step 1- When a patient is brought to the hospital for treatment sensor nodes are attached to his body. These sensors measure the vital signs of the patient body like the heart rate, temperature or blood pressure etc. All the sensor node attached to the body area network of the patient which receives data and forwards it to the gateway node.

Step 2- The Transmit and Receive (T\&R) technique use Clustering to gather sensor information from sensor nodes to gateway or sink node either it may be in range or may not be in range from the sensor network. When the sink node out of range from other sensor network it uses the Clustering techniques where Cluster Head is responsible to communicate with the with the sink node thorough the intracluster network or intercluster network. Actually, in this method the transmit node and receive node are included in the same node.

Step 3- Each sensor node attached to the patient's body area network has a unique ID through which it can be uniquely identified. All the sensors used are wireless and Zigbee technology is used for communication between them and their cluster heads. The cluster heads also use Zigbee technology to forward their data to the sink directly or through other cluster heads.

Step 4- In a Wireless Sensor Network after sensing, the sensed data like BP sensor, ECG sensor, EMG Sensor, Body positioning sensor etc. is stored in a cloud environment where the data is always available. After gathering data into Gateway node few data processing may appear locally and after that the data is dispatched to the server through the internet and saved into Database for future use. The authorized remote and local user can access those data through the internet connectivity.

Step 5- The sink is a computer in the sensor network used to establish a connection with outside world. Doctors analyse vital signs which are stored in a database and take necessary actions. Based on each patient's health condition clinician/doctor fixes a threshold for each sensor data that is received from the sensor attached to the patient. The sink then transmits these threshold values to that patient's cluster head. The cluster head then forwards each threshold to its respective sensor node. Each threshold is then stored in its respective sensor until its again updated by the health monitor.

Step 6- After a new threshold is received by a sensor node it compares its current readings with the threshold. If the current readings deviate significantly from the threshold then that sensor node forwards its readings to the cluster head which then forwards it to the sink node. The sink node receives and sends the readings to the server which has deviated from their thresholds after which the clinicians take the necessary actions.

Step 7- During transformation of data to the server the field will be as shown in Table I below:-

Table I. Field in a Packet Data Unit

\begin{tabular}{|c|c|c|c|}
\hline $\begin{array}{c}\text { Sink/ } \\
\text { gateway } \\
\text { Address }\end{array}$ & $\begin{array}{c}\text { Dest. Address } \\
(172.16 .254 .1)\end{array}$ & $\begin{array}{c}1.2 .3 \text { (sensor } \\
\text { address) }\end{array}$ & $\begin{array}{c}\text { Temperatu } \\
\text { re Data }\end{array}$ \\
\hline $\begin{array}{c}2.2 .3 \\
\text { (sensor } \\
\text { address) }\end{array}$ & BP Data & $\ldots \ldots \ldots . .$. & Checksum \\
\hline
\end{tabular}


Step 8- For data acquisition from sensor nodes, Sensor network uses a Sensor Gateway, which helps to store data into the Data server through the Internet service. In the Data server there is Database where various attribute of data is stored and they can be used at any time remotely.
Step 9- For data consumption from the data server, the clients are using browser application which will help them to stay connected to the data server through the Internet. In Data Server there is running a server application like App Server and it gives result to the clients (doctors, caregiver) after requesting to the Database.

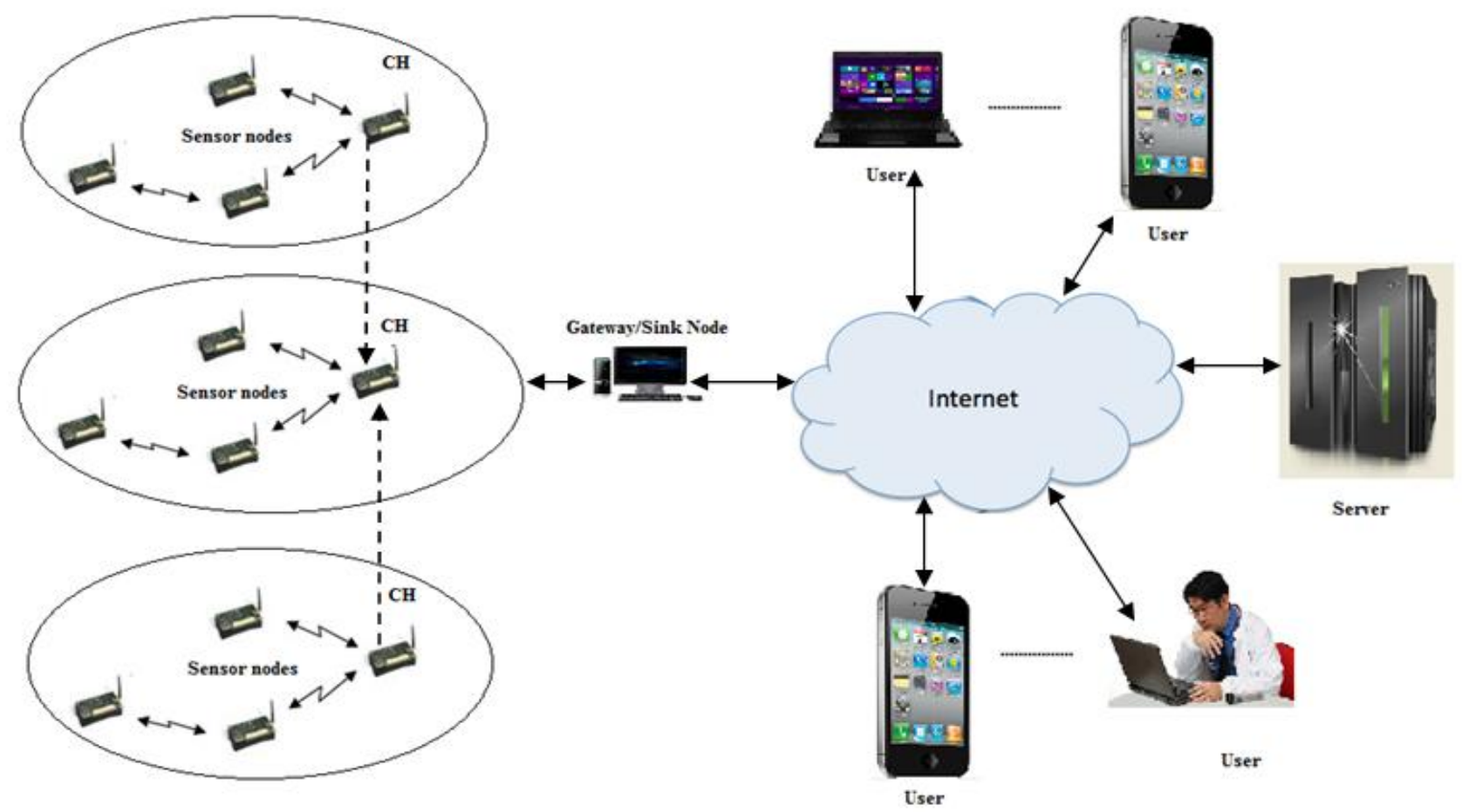

Fig 1: Components of WSN Monitoring System

\section{THE TRANSMIT AND RECEIVE}

\section{TECHNIQUE:}

\subsection{Transmit and Receive Clustering}

\section{Technique}

In Fig 2, to form a cluster in the network eventually depends upon the level-two hierarchy in which the Cluster Head nodes are used to make the hierarchy of the upper level and the other member of the cluster network nodes used to create lower level of the hierarchy. The sensor nodes send data continuously after some time to their respective Cluster Head $(\mathrm{CH})$ nodes [13]. These Cluster Head nodes assemble all the incoming data minimizing the number of sending packets and dispatched these data directly to the Sink node or through the intermediate cluster head nodes to communicate with the sink node. The cluster head has a big task to send data to the sink node. $\mathrm{CH}$ always sends data to a long distance then the any other common sensor nodes, so they assuredly expend more power. It periodically re-selects the $\mathrm{CH}$ among all the presented nodes in each cluster and the role of $\mathrm{CH}$ will be divided among all the present nodes and saves energy.

\subsection{Transmit Stage and Receive Stage}

In data collection there are two separate phases one is Transmit stage the other is Receive stage. In this method the sink node transmits the threshold data which is set by the

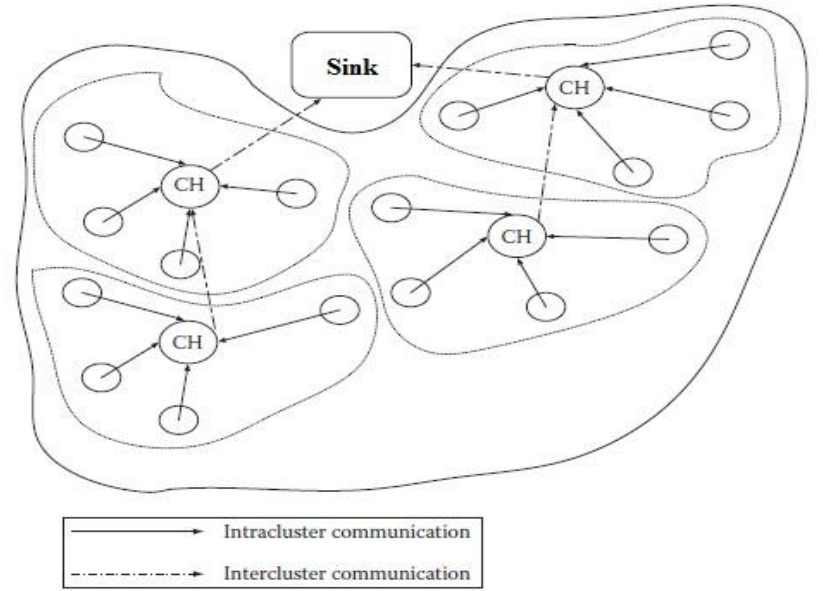

Fig 2: Wireless Data Communication in Clustered Network

doctor after analysing the patient's current situation stored in the database and receives the data from the sensor node.

- Transmit stage: In the Transmit stage, doctor sees the database and fixes a threshold and this threshold is transmitted by the sink computer 
to their corresponding cluster network using their respective IDs.

- Receive stage: During the receive stage the sensed information is remitted by wireless sensor nodes to the sink or gateway node. In the receive stage, if the others nodes are out of range from the sink node in this case the $T \& R$ with clustering technique is used, where the nodes in the network launch continuously their reading to their respective Cluster $\operatorname{Head}(\mathrm{CH})$ nodes. The $\mathrm{CH}$ nodes put together all the incoming data and compares with the new threshold value send by the sink node and dispatch them directly to Sink node or through the intermediate cluster head nodes to communicate with sink node. Each sensor node and the cluster head sends data in this technique to reduce the communication over head and reduces the power utilization.

The state transition between the sink node, transmit node and wireless sensor nodes are shown below in Fig 3 The position of the transmit and sink node transfers periodically between receive, analyse \& set value, and transmit. The sink node gathers the sensed information of sensor nodes after getting the new threshold by the transmit node and saves it in to the sensor database of sensor nodes. After getting the transmit information the wireless sensor node sense the information and compare it after that dispatch the related information to the sink node.
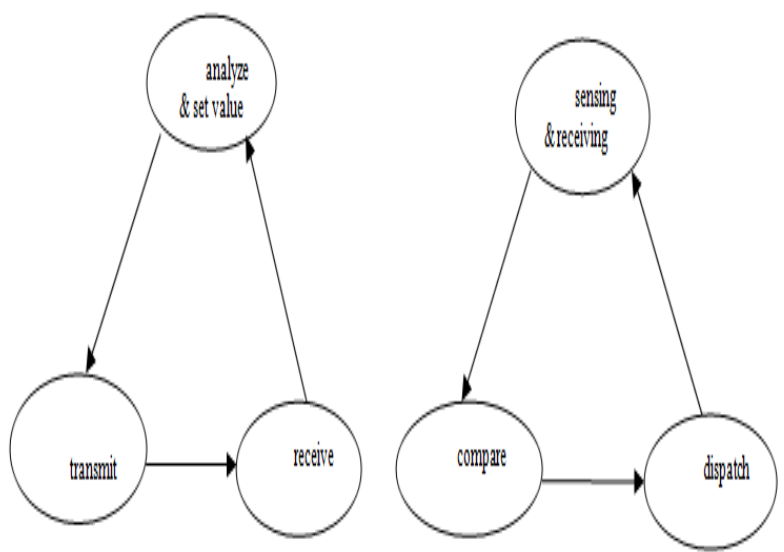

Fig 3: State Diagram for T\&R Using Clustering Method

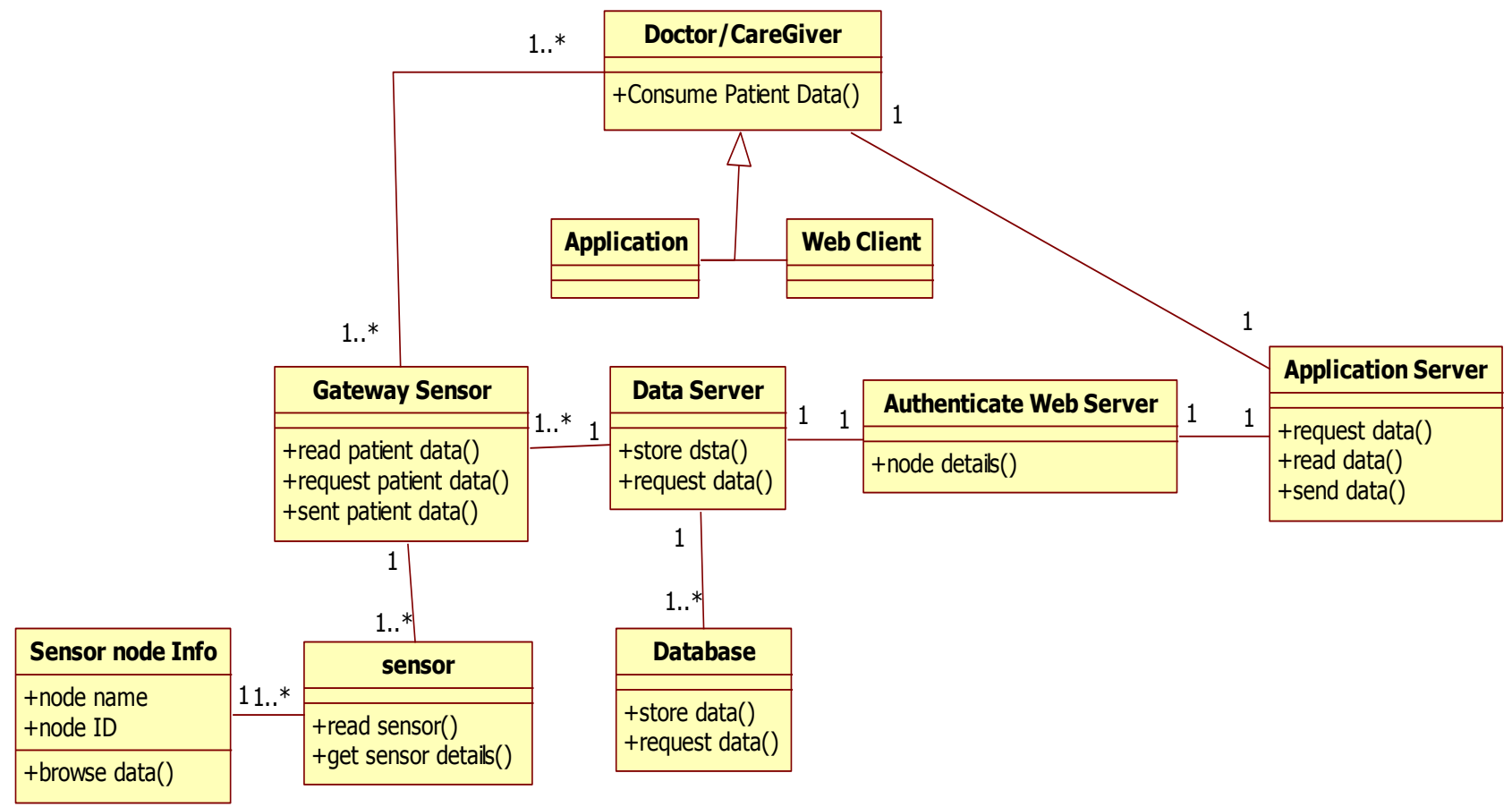

Fig 4: Class Diagram for WSN Data Monitoring System

The above Fig 4. Shows the Class diagram for the Wireless Sensor Networks monitoring system. In a network there may be many sensor nodes attached to a gateway node. For data acquisition, all the information from sensor nodes comes to sensor gateway. The sensor gateway sends this data through internet to a data server then this data is saved in the database where these data can be accessed remotely any time. And for data consumption, the Doctor or Caregiver has a web application or application which will help him to establish a connection with data server. The data server stores the data in Database and in data server there is a server application it will help the doctors or caregivers by giving the appropriate information queried from database. 


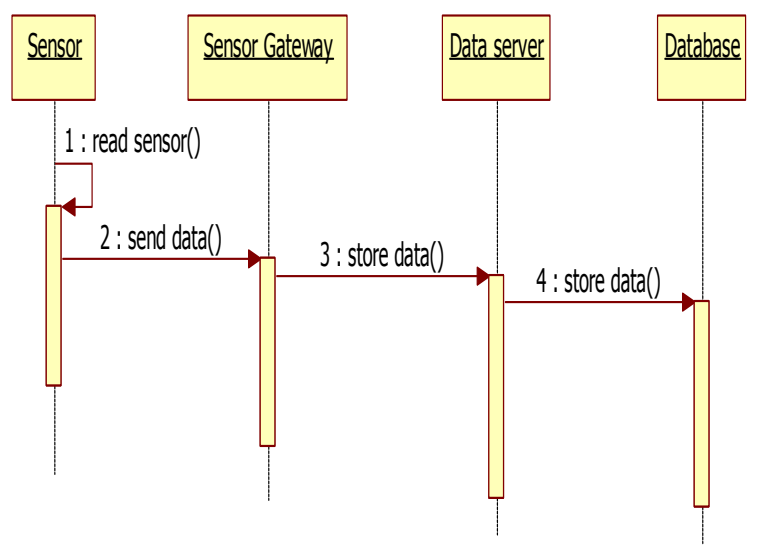

Fig 5: Sequence Diagram for WSN Data Acquiring and Storage

Figure 5. shows the data Acquiring method the sequence diagram. The occurring steps is as follows:-

- Firstly, the Sensor node in the WSN reads the information continuously.

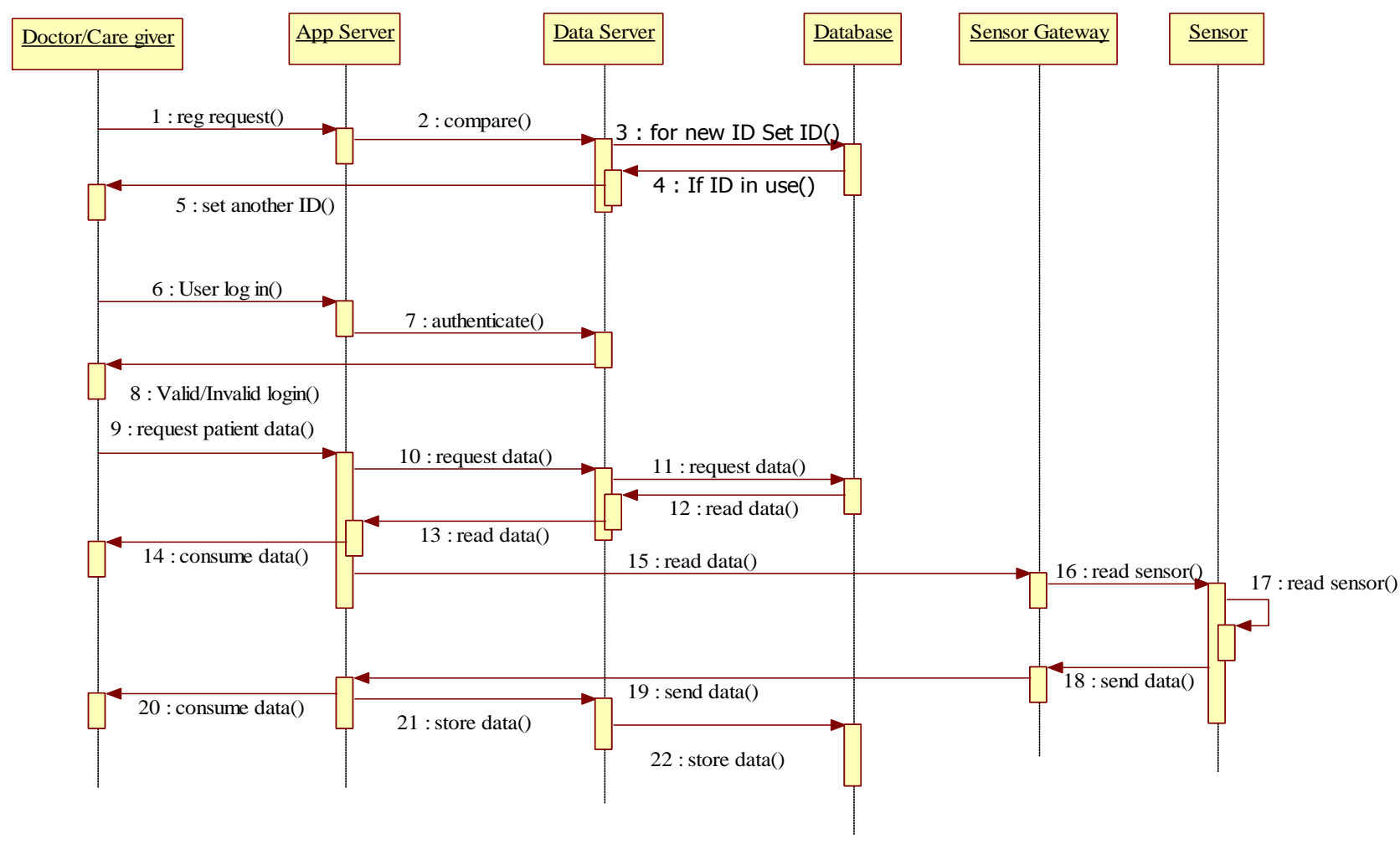

Fig 6: Sequence Diagram for Data Consumption

5. After getting authorization the doctor or caregiver can request to application server for patient data. Then application server can requests to data server and data server requests to database and it sends the appropriate information to Application Server and it sends those data to the user.
- Then the Sensor sends the data to their Gateway node.

- After that the Gateway node transmits the data to the Data Server, before sending data the Sensor gateway may compute those data.

- Then the Data Server saves the data in the Database.

Fig 6. in below shows the data Consumption method the sequence diagram.

1. Firstly, doctors or caregiver sends registration request to application server.

2. Then application server sends request for Identification no to data server and the Id is new for system then it can accept registration request.

3. If there is same ID stored in data server, data server gives a message ID IN USE () and tells to set another ID ().

4. So, to access Database, user should be authenticating. To verify authentication the user must follow authentication phase in data server.

6. For first time when there is no data is available in the database, application server sends the read data requests to the gateway node or Sensor Gateway.

7. Gateway node sends a message called read data () to Sensor nodes of this network. 
8. The Sensor node takes the reading of the patient attached to his body.

9. The Sensor transmits the sensed data to the Sensor Gateway.

10. The Sensor Gateway may compute some tasks of this collected data in local machine and dispatches the data to the Application Server.

11. The Application Server gives these data to the requested person and also send to data sever for storage in Database.

\section{CONCLUSION AND FUTURE WORK}

ZigBee technology has many advantages like it is a low power ,close range, low cost, simple, technology and it is based on IEEE802.15.4 standard. In the Zigbee network there may be thousands of small sensor nodes coordinate with one another to build a communication network. Therefore, it is scalable. Additionally data collected at base stations from different sensor nodes is forwarded to other networks such as WLANs and cellular networks. So the sensor data sent over to other networks must be globally compatible. The data format used should also be good enough such that the transmission of large volumes of data is possible and should not drain the sensor battery or overload the processor of the sensor. Therefore, here proposed a data format for transmission in WSNs that will be globally compatible with WLANs and cellular networks so that it can be used in a cross layer framework consisting of heterogeneous devices and also for data collection $\mathrm{T} \& \mathrm{R}$ method with clustering technique gives huge advantages to this proposal .

In future author plan to implement this framework in a real time environment and test other module of communication and other packet formats which are compatible with zigbee and see if overheads can be further reduced.

\section{REFERENCES}

[1] Pottie, G.J. ; Dept. of Electr. Eng., California Univ., Los Angeles, CA, USA "Wireless sensor networks",Information Theory Workshop, 1998,pp. 139 140, 22-26 Jun 1998

[2] Puccinelli, Daniele. and Haenggi, Martin. "Wireless sensor networks: applications and challenges of ubiquitous sensing", Circuits and Systems Magazine, IEEE (Volume: 5, Issue: 3), pp. 19 - 31, 2005.

[3] Akyildiz , I.F. W. Su, Sankarasubramaniam, Y. and Cayirci, E. "A survey on sensor networks," in IEEE Communications Magazine,pp. 102-114, Aug. 2002.
[4] Rajkumar, Luke. "Remote Patient Monitoring and Healthcare Applications".

[5] Fujita,Yoshihisa, T. ; Tsukamoto, M. "A Sensing System for Data Collection with Broadcasting Predicted Data" Intelligent Sensors, Sensor Networks and Information Processing, 2008. ISSNIP 2008. International Conference on, pp. 465 - 470, 15-18 Dec. 2008.

[6] Erratt, N. ,Yao Liang" The Design and Implementation of A General WSN Gateway for Data Collection" Wireless Communications and Networking Conference (WCNC), 2013 IEEE, pp. 4392 - 4397, 7-10 April 2013.

[7] Singh, Dhananjay., Tiwary, U.S., Lee, Hoon-Jae., WanYoung Chung "Global Healthcare Monitoring System using 6lowpan Networks" 11th International Conference on Advanced Communication Technology (ICACT2009), phoenix park, Korea, pp.113-117, Feb.2009.

[8] Madden., S. R., Franklin., M. J., and W. Hong, "Tinydb:an acquisitional query processing system for sensor networks," ACMv Trans. Database Syst., vol. 30, no. 1, pp. 122-173, 2005.

[9] Erratt., N. and Liang., Y., "Compressed data-stream protocol: an energy efficient compressed data-stream protocol for wireless sensor networks," Communications, IET, vol. 5, pp. 2673-2683, 2011.

[10] Marcus., Anthony., Cardei., Mihaela, Cardei., Ionut,"A pattern for web-based wsn monitoring ", journal of communications, vol. 6 , no. 5, august 2011.

[11] He., Dayu., "The ZigBee Wireless Sensor Network in Medical Care Applications", Computer, Mechatronics, Control and Electronic Engineering (CMCE), 2010 International Conference on ,Vol:1, pp. 497 - $500,24-26$ Aug. 2010

[12] Pattichis C. S., Kyriacou E., Voskarides S., Pattichis M. S.,Istepanian R., Schizas C. N. "Wireless Telemedicine Systems: An Overview[J]." Antennas and Propagation Magazine, 2002, 44(2):143-153.

[13] Mamalis., B, Gavalas D, Konstantopoulos., C, and G. Pantziou,"Clustering in Wireless Sensor Networks," RFID and Sensor Networks: Architectures, Protocols, Security and Integrations, Y. Zhang, L.T. Yang, J. Chen, eds., pp. 324-353, CRC Press, 2009.

[14] Guestrin .Carlos, Joseph M. Hellerstein, Wei Hong, Deshpande ., Amol, R. Madden ., Samuel " ModelDriven Data Acquisition in Sensor Networks" Proceedings of the 30th VLDB Conference,Toronto, Canada, 2004. 